\title{
THE ROLE OF ERUPTION IN SOLAR FLARES
}

\author{
PETER A. STURROCK \\ Center for Space Science and Astrophysics, Stanford University, Stanford, CA 94305, U.S.A.
}

\begin{abstract}
This article focuses on two problems involved in the development of models of solar flares. The first concerns the mechanism responsible for eruptions, such as erupting filaments or coronal mass ejections, that are sometimes involved in the flare process. The concept of 'loss of equilibrium' is considered and it is argued that the concept typically arises in thought-experiments that do not represent acceptable physical behavior of the solar atmosphere. It is proposed instead that such eruptions are probably caused by an instability of a plasma configuration. The instability may be purely MHD, or it may combine both MHD and resistive processes. The second problem concerns the mechanism of energy release of the impulsive (or gradual) phase. It is proposed that this phase of flares may be due to current interruption, as was originally proposed by Alfvén and Carlqvist. However, in order for this process to be viable, it seems necessary to change one's ideas about the heating and structure of the corona in ways that are outlined briefly.
\end{abstract}

\section{Introduction}

There appear to be several types of solar flares (Bai and Sturrock, 1989), ranging from simple $\mathrm{X}$-ray and $\mathrm{H} \alpha$ brightenings to the complexity of large two-ribbon flares. It appears that flares with high-energy manifestations, such as gamma-ray emission, tend to involve eruptive mass motion (see, for instance, Bai, 1986a). It has been argued (Kahler, 1982) that such an association may be due simply to the 'big flare syndrome', namely, that everything that can happen in a flare will happen in a big flare. However, the association between eruptive events and high-energy events in flares does raise legitimate questions, of which we here consider only two.

(1) What is the mechanism that leads to an eruption such as a coronal mass ejection? And

(2) Is there any physical reason why an eruption should lead to a high-energy event? We consider these questions in the next two sections.

\section{Loss of Equilibrium}

In recent years, it has been proposed by several authors that eruptions in solar active regions, that might give rise to CMEs, may be ascribed to a concept termed 'loss of equilibrium'. This concept arises in the study of force-free magnetic-field configurations of translational symmetry, that may be described in terms of a 'generating function'.

Consider a magnetic-field configuration that is uniform in the $z$-direction of Cartesian coordinates $z, x, z$. Since $\nabla \cdot B=0$, we see that the magnetic field may be expressed as

$$
\mathbf{B}=\left(\frac{\partial A}{\partial y},-\frac{\partial A}{\partial x}, B_{z}\right)
$$

Solar Physics 121: 387-397, 1989.

(C) 1989 Kluwer Academic Publishers. Printed in Belgium. 
We then find that the condition

$$
\mathbf{j} \times \mathbf{B}=0
$$

for the field to be force-free is satisfied if

$$
\nabla B_{z} \times \nabla A=0
$$

which implies that $B_{z}$ is expressible as a function of $A$, and if

$$
\nabla^{2} A+B_{z} \frac{\mathrm{d} B_{z}}{\mathrm{~d} A}=0
$$

So far, the treatment is quite general, except that we are requiring translational symmetry.

At this point, we note that it is possible to define a family of solutions of Equation (2.4) by assuming that

$$
B_{z}=\lambda F(A) \text {. }
$$

Then Equation (2.4) becomes

$$
\nabla^{2} A=-\lambda^{2} f(A)
$$

where

$$
f(A)=F(A) F^{\prime}(A) .
$$

Equation (2.6) describes a family of force-free magnetic-field configurations of translational symmetry, corresponding to varying values of the parameter $\lambda^{2}$, for a given form of the generating function $f(A)$.

As a specific example, we consider the case discussed originally by Low (1977a) and later by Birn, Goldstein, and Schindler (1978) and Priest and Milne (1980). In our notation, the generating function is given by

$$
f(A)=-k^{2} \exp (-2 A) .
$$

The magnetic flux $B_{y}$ at the plane $y=0$, that represents the photosphere, is given by

$$
A(x, 0)=\ln \left(1+k^{2} x^{2}\right) .
$$

Low shows that Equation (2.6) and the boundary condition (2.9) are satisfied by the function

$$
A(x, y)=\ln \left[1+k^{2} x^{2}+2\left(\frac{1-\mu^{2}}{1+\mu^{2}}\right) k y+k^{2} y^{2}\right],
$$

where $\lambda$ and $\mu$ are related by

$$
\lambda=\frac{4 \mu}{1+\mu^{2}} .
$$




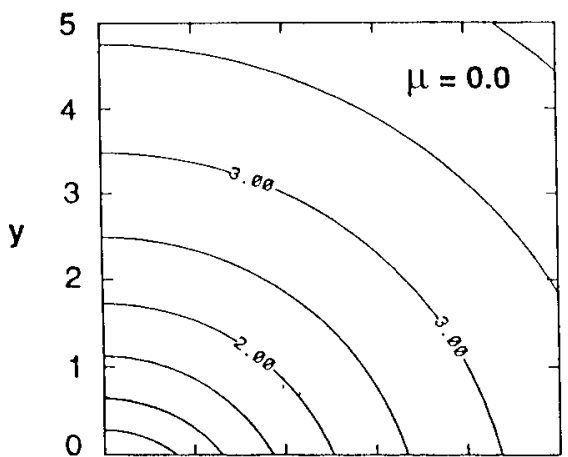

(a)

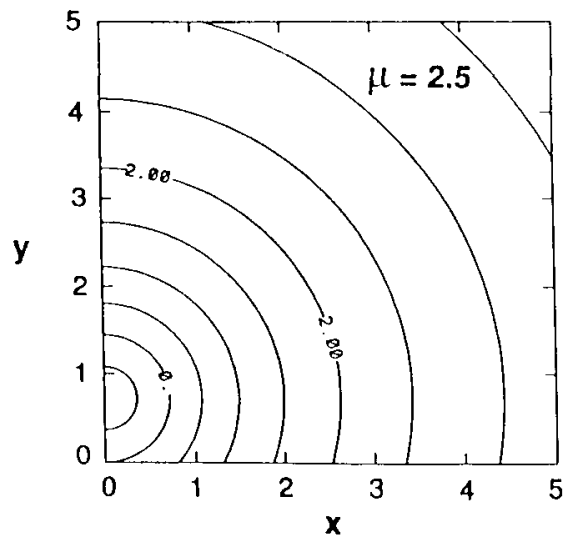

(c)

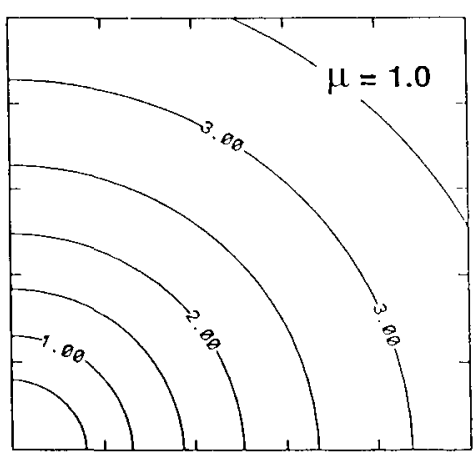

(b)

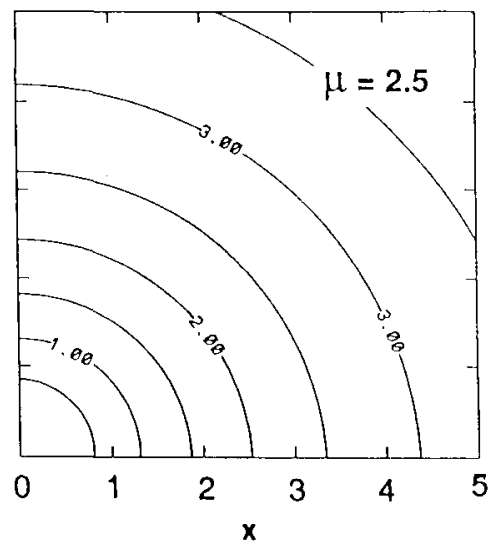

(d)

Fig. 1. End-on view of magnetic field lines for the model defined by Equation (2.10). (a), (b), and (c) are taken from the generating-function model for the values $\mu=0, \mu=1$, and $\mu=2.5$. It is seen from (c) that for $\mu>1$ this model involves floating flux. (d) shows the magnetic-field configuration that arises from numerical solution of the force-free-field equations with the same boundary conditions as case (c). It is seen that it is indeed possible to meet those boundary conditions without the introduction of floating flux.

Low considers the sequence of magnetic-field configurations formed by allowing $\mu$ to increase from 0 to $\infty$. Then $\lambda$ increases from 0 to a maximum of 2 (when $\mu=1$ ) and then decreases back down to 0 . For $0<\mu \leq 1$, the magnetic-field configuration is that of a simple arcade (see Figures 1(a) and 1(b)) in which the distribution of footpoints on the photosphere is given by

$$
k z=\frac{2 \mu}{1+\mu^{2}} \arcsin \left\{\left[\left(\frac{1-\mu^{2}}{1+\mu^{2}}\right)^{2}+k^{2} x^{2}\right]^{-1 / 2} k x\right\} .
$$

For $\mu>1$, the magnetic-field configuration is no longer that of a simple arcade. It contains a flux tube that runs above and parallel to the photosphere, that may be termed 
'floating flux' (see Figure 1(c)). The distribution of footpoints is now given by

$$
k z=\frac{2 \mu}{1+\mu^{2}}\left(\pi \frac{x}{|x|}-\arcsin \left\{\left[\left(\frac{1-\mu^{2}}{1+\mu^{2}}\right)^{2}+k^{2} x^{2}\right]^{-1 / 2} k x\right\}\right) .
$$

Low takes the position that the creation of floating flux is forbidden by the assumption of infinite electrical conductivity, and concludes that 'field configurations with $\mu>1$ are not available to the evolving magnetic field'. He suggests that the quasi-steady evolution of the force-free field ceases at $\mu=1$, whereupon explosive events take over.

The question we need to address is whether the physical problem represented by this mathematical model is relevant to processes that can occur in the Sun's atmosphere. For the range of parameters $\mu \leq 1$, the problem is equivalent to that of moving a prescribed distribution of magnetic flux according to the displacement given by Equation (2.12). This is a physically acceptable thought experiment.

On the other hand, for $\mu>1$, the evolution of the sequence, that requires the global constraint of the functional relationship between $B_{z}$ and $A$ described by Equations (2.5), (2.7), and (2.8), is not equivalent simply to the footpoint motions described by Equation (2.13). As Low points out, it requires also the introduction of flux not connected to the photosphere. Klimchuk and Sturrock (1989) therefore argue that, for $\mu>1$, the generating function problem defined by Low does not specify a physically acceptable thought experiment. This being the case, consequences of that thought experiment are not relevant to the actual behavior of the Sun's magnetic field.

Indeed, using the magneto-frictional method developed by Yang, Sturrock, and Antiochos (1986), we have been able to calculate force-free magnetic-field configurations corresponding to the continued evolution of the footpoints through Equation (2.12) for $0<\mu \leq 1$, and then through Equation (2.13) for $\mu>1$. We find that the magnetic field develops in a well-behaved manner and shows no evidence of catastrophic behavior (see Figure 1(d)).

As a result, we have concluded that the concept of 'loss of equilibrium' is an artifact of the specification of physically unacceptable thought experiments. Hence the concept cannot be invoked as an explanation of magnetic-field eruptions related to flares and CMEs.

In the above calculations, we have considered strictly force-free magnetic-field configurations. In reality, the coronal magnetic field is always interacting with plasma of non-zero density and pressure, so that the field is never exactly force-free. In articles following the one previously discussed (Low, 1977a), Low (1977b, 1980) has considered the implications of non-zero plasma pressure upon the evolution of a sequence of magnetic-field configurations defined by a particular choice of generating function. By using the Bernstein integral to test for MHD stability (Bernstein, 1973a, b), Low finds, in terms of his parameter $\lambda$, that the system is stable for $\lambda<\lambda^{*}$, where $\lambda^{*}$ is the critical value of $\lambda$, but that it is only marginally stable for $\lambda=\lambda^{*}$. Hence Low conjectures that, in a system with non-zero gas pressure, an approach to the 'loss-of-equilibrium' state (as previously defined) is also an approach towards MHD instability. If this conjecture 


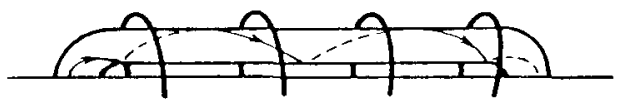

(a)

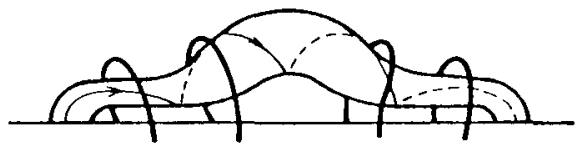

(b)

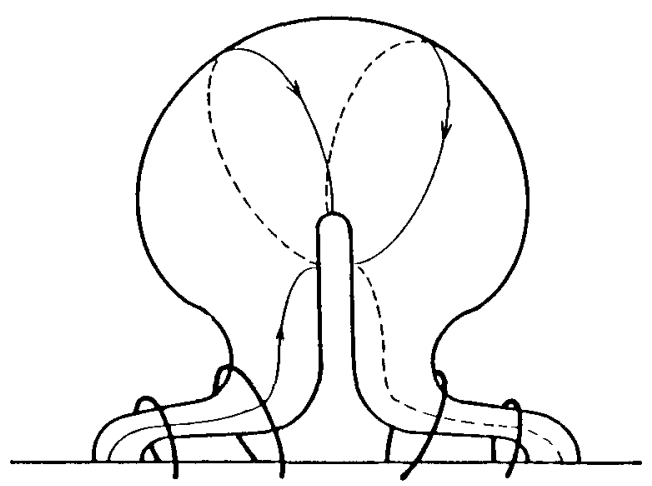

(c)

Fig. 2. A possible MHD interpretation of solar eruptions. (a) shows a twisted flux tube anchored at both ends and held down by an overlying magnetic arcade. (b) indicates how the flux may begin to emerge by displacing selected field lines of the arcade. (c) shows that the flux tube would unwind in the region held down by the arcade, transferring most of the twist to the part that is erupting. If the original flux tube is long enough and sufficiently twisted, the initial state will have higher magnetic energy than the final state of a completely open configuration.

can be shown to have general validity, then the concept of loss of equilibrium would acquire general physical significance. However, this general connection between loss of equilibrium and MHD instability has so far not been demonstrated.

If magnetic-field eruptions are not due to 'loss of equilibrium', this leaves us with the problem of finding an alternative interpretation. The most likely explanation is that the eruption is due to some type of instability.

One possibility is that the instability is purely MHD in nature. For instance, consider the configuration shown schematically in Figure 2, comprising the horizontal flux tube rooted in the photosphere at its ends and held down by an overlying magnetic arcade. 


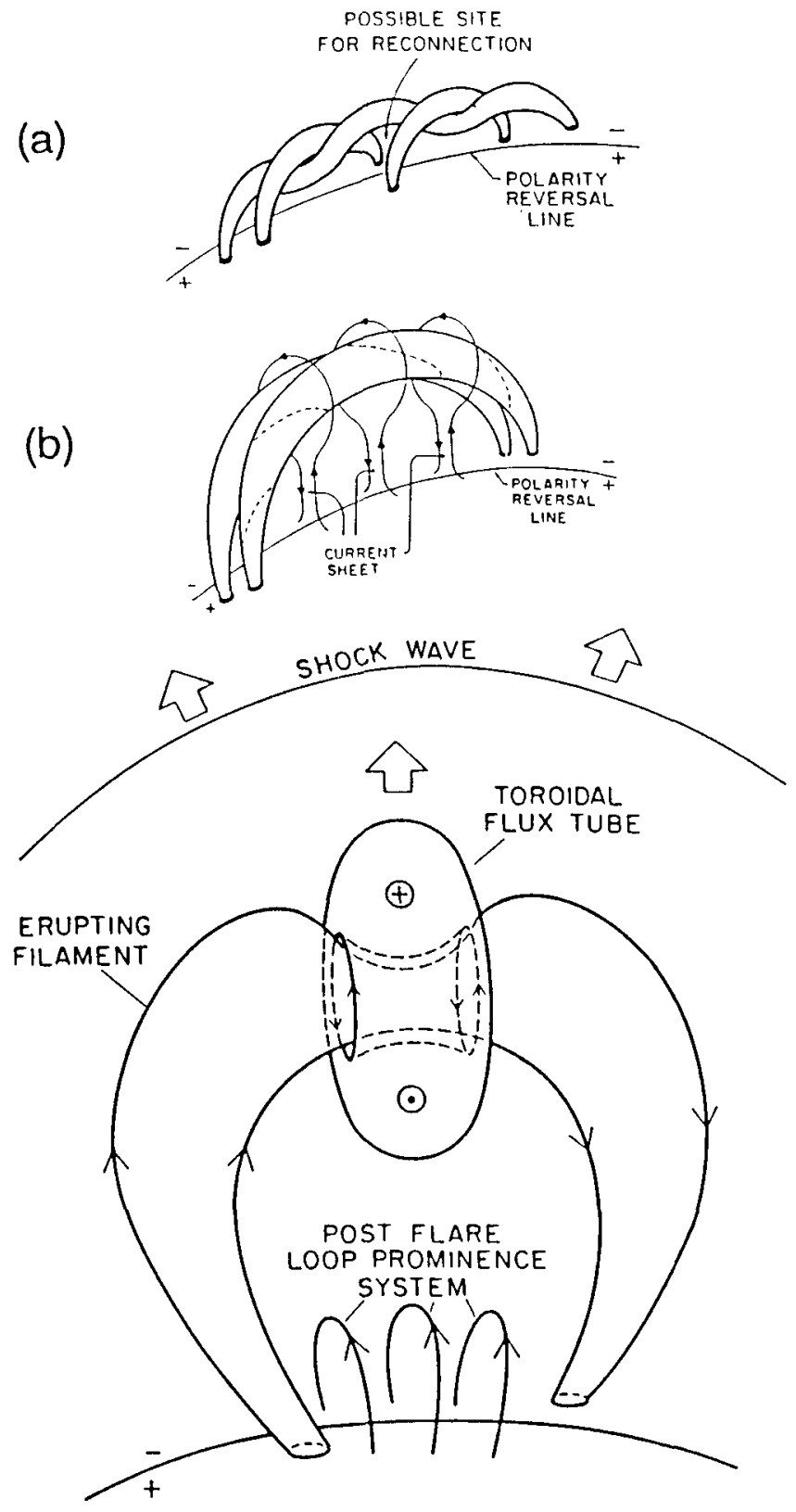

(c)

Fig. 3. A possible interpretation of solar eruptions that involves both MHD and resistive processes. (a) shows a schematic representation of a possible magnetic configuration associated with a filament, that of a magnetic rope comprising a number of magnetic strands. Reconnection can occur where strands of opposite polarity are in contact with each other. (b) is a schematic representation of the evolution of the magnetic system after all connection with the photosphere has been broken, except the connection at the ends of the erupting flux tube. As a result of this eruption, magnetic field lines of the arcade form a new current sheet below the erupting flux tube. (c) is a schematic representation of the final form of the magnetic 
If the flux tube is sufficiently long and sufficiently twisted, an eruption of the flux tube, corresponding to a rupture of the arcade, would be energetically favorable. This is the case since the tube can now move towards an untwisted state, so reducing the magnetic energy along its entire length. By making the tube long enough, and sufficiently highly twisted in its initial state, the energy so released can certainly exceed the finite energy of the open-field configuration that would be the limiting state after the eruption has occurred. An estimate of the critical condition for instability could be made either in terms of the small-amplitude energy theorem of Bernstein (1973a, b) or by calculating the initial and final magnetic field configurations and comparing their energies. (It should perhaps be emphasized that we are proposing that only part of the magnetic flux erupts into the open state. Hence there is no conflict between this proposal and the conjecture advanced by Aly (1984), that the completely open field is the state of maximum energy for a magnetic field with prescribed values of the normal field on the bounding surface.)

The above proposal represents a purely MHD instability. By contrast, it is possible that the instability responsible for eruptions involves both MHD and resistive processes. For instance, it may be more realistic to consider the initial state of a filament as being comprised of many individual strands, as shown in Figure 3(a). Reconnection can occur between adjacent strands of opposite polarity. This has two effects. One is that the reconnection would give rise to energy release that could lead to soft $\mathrm{X}$-ray emission and $\mathrm{H} \alpha$ emission. The other consequence is that the remaining strands would be increasingly stressed. This may lead to runaway reconnection that then leads to the formation of a flux tube rooted in the photosphere only at its ends, as shown in Figure 3(b). This flux tube will be twisted. If the twist is sufficiently great, the tube would erupt towards an open configuration as shown in Figure 3(c).

\section{Magnetic-Field Eruption and Particle Acceleration}

As noted some time ago by Kopp and Pneuman (1976) and by Anzer and Pneuman (1982), the eruption of a filament will produce an important change in the topology of the magnetic arcade that overlays the filament before the eruption. This is shown schematically in Figure 3. The topology is that of an open bipolar magnetic-field configuration, that was proposed some time ago (Sturrock, 1968) as being a plausible explanation of two-ribbon flares. According to the preceding authors, reconnection at the current sheet separating the two parts of the open bipolar flux system results in energy release that can explain the long-lived soft X-ray emission and also the $\mathrm{H} \alpha$ emission. Cliver et al. (1986) point out that this process may, on occasion, also give rise to weak hard $\mathrm{X}$-ray emission.

We have learned from observations of hard X-ray and gamma-ray emission from flares that particle acceleration, leading to nonthermal events, typically occurs during

field when the original field is sufficiently stressed that it results in ejection leading to an open configuration. Reconnection of the extended current sheet can now lead to the formation of a toroidal flux tube surrounding the magnetic field of the erupting filament. 
the 'main' energy-release phase of solar flares (Bai, 1986b; Bai and Sturrock, 1989), a term that is used to include the impulsive and the gradual nonthermal phases of energy release. It has been proposed elsewhere (Sturrock et al., 1984) that this phase may be attributed to reconnection at the current sheets separating elementary flux tubes of an active region. It certainly is reasonable to look to reconnection as an explanation of energy conversion, since the release of free magnetic energy requires a change in the connectivity of magnetic-field lines. However, it is not clear that tearing-mode-type reconnection can accelerate sufficiently large numbers of particles sufficiently rapidly to explain the short time-scale of particle acceleration that has become apparent from SMM observations (Kane et al., 1986).

Another puzzle concerning flare behavior is the relationship between eruptions and the main phase of flares. It is well known that the eruption normally begins well before the main phase (Martin and Ramsey, 1972). This suggests that the eruption somehow leads to conditions that trigger an instability that is manifested as the main phase of a flare. We have suggested elsewhere (Sturrock, 1987, 1988) that the Kelvin-Helmholtz instability may develop in the neighborhood of an erupting filament when the speed attains a critical value, and that this instability may develop MHD turbulence that then speeds up the tearing-mode reconnection process in existing current-carrying magneticfield systems. We now discuss another possible interpretation of the relationship between eruptions and the main phase of flares.

It was pointed out some time ago by Alfvén and Carlqvist (1967) that energy release in a flare may be related to current interruption. If, in a twisted magnetic flux tube, the resistivity were suddently to increase in a localized region, a strong electric field must develop in that region to maintain the current. Such a current must persist until the field can unwind, a process that requires Alfvén-wave propagation, so that the acceleration must persist for the Alfvén-wave propagation time.

In their article, Alfvén and Carlqvist suggest that the relevant instability is the simple two-stream electron-ion instability. For the case of an isothermal electron-proton plasma, the condition for instability (Krall and Trivelpiece, 1973) is that

$$
v_{d}>1.3\left(k T / m_{e}\right)^{1 / 2} \approx 10^{5.7} T_{e}^{1 / 2} .
$$

On noting that the relative drift velocity of the electrons and protons is given by

$$
j=n c^{-1} e v_{d},
$$

and that the magnitude of the current is given by

$$
|j| \approx \frac{1}{4 \pi} B b^{-1}
$$

where the magnitude of the curl of the magnetic field is taken to be determined by the length scale $b$, we find that the condition for instability becomes

$$
n b<10^{13.0} B T_{e}^{-1 / 2} .
$$


For values typical of the coronal component of an active region $T=10^{6.4}, n=10^{9}$, and $B=10^{2}$, we find that this instability will occur only if $b<10^{2.8}$, i.e., if the scale of the current system is less than about $6 \mathrm{~m}$. Even if this were to occur, clearly such a region would involve only a very small fraction of the volume of the magnetic-field configuration, and therefore lead to the release of only a very small fraction of the total free magnetic energy. It therefore seems unlikely that, in such conditions, current interruption will play a significant role in energy release.

We now suggest two changes in this model that make the idea more attractive. First, we may note that the ion-acoustic instability sets in at a lower critical value of the current density, provided the electron temperature is substantially higher than the ion temperature. If the electron temperature is substantially higher than the proton temperature, the condition for instability (Krall and Trivelpiece, 1973) is

$$
v_{d}>\left(k T_{i} / m_{i}\right)^{1 / 2} \approx 10^{4.0} T_{i}^{1 / 2} .
$$

Hence the condition (3.4) is now replaced by

$$
n b<10^{14.7} B T_{i}^{-1 / 2} .
$$

The coronal conditions considered above now require that $b<10^{4.2}$ for instability. This is still a very small value so that the objection made above is still applicable.

Our second change in the model requires a departure from the conventional interpretation of the mechanism that maintains the solar corona, that there is a steady heating mechanism that maintains the corona at a temperature of about $10^{6} \mathrm{~K}$. In recent years, some attention has been given to an alternative possibility that the corona is heated only by localized flare-like energy releases (see, for instance, Blake and Sturrock, 1985; Parker, 1988). For instance, it may be appropriate to regard the corona as composed of many small filaments, each filament comprising a small flux tube rooted at both ends in the photosphere. Sudden energy release in a small tube will lead to chromospheric evaporation, so that the tube is soon filled with plasma at a temperature of order one million degrees. However, this gas will cool by radiation and by conduction, tending to acquire the same temperature as the chromosphere.

This assumption has two consequences. The first is that the temperature to consider in Equations (3.4) and (3.6) may be nearer $10^{4} \mathrm{~K}$ than $10^{6} \mathrm{~K}$. The second consequence is that the lower temperature of the gas leads to a lower scale height, so that the density may be very much lower than that we normally ascribe to the corona in an active region.

Consider, as an example, that the temperature in a loop is in fact of order $10^{4} \mathrm{~K}$, but $T_{e}$ is higher than $T_{i}$. For instance, consider the case that $T_{e}=10^{4.5}$ and $T_{i}=10^{4.0}$. Also consider an elementary flux tube with radius of order $10^{8} \mathrm{~cm}$, corresponding to a flux of $10^{18.6} \mathrm{Mx}$ if $B=10^{2} \mathrm{G}$. Adopting $b=10^{8}$, we now find that the ion-acoustic instability sets in if $n<10^{6.7}$. However, for the temperatures quoted, the scale height is only $10^{8.0} \mathrm{~cm}$, so that the density will drop from the chromospheric value of $10^{12} \mathrm{~cm}^{-3}$ to the required value of $10^{6.7} \mathrm{~cm}^{-3}$ in 12 scale heights, that is, in about $10^{9.1} \mathrm{~cm}$ or about $13000 \mathrm{~km}$.

We see that, with these revised assumptions, current interruption could very well 
occur in an active region so that it may indeed play a role in the main phase of solar flares, as suggested by Alfvén and Carlqvist.

We can now come back to the question posed in the Introduction: "Is there any physical reason why an eruption should lead to a high-energy event?" If the main phase may indeed be attributed to current interruption by the ion-acoustic instability, we need to inquire whether an eruption could lead to a sudden reduction in the plasma density and/or a sudden increase in the ratio of the electron temperature to the ion temperature. The answer to both these questions appears to be in the positive.

As we saw in Section 2, the magnetic arcade above an erupting filament will be displaced vertically as a result of the eruption. Hence the density at the top of an arcade loop will decrease. The density could therefore drop to the critical value for ion-acoustic instability. Note also that this interpretation provides a simple justification for the common assumption that acceleration occurs at the top of a loop.

However, in order for the ion-acoustic instability to occur, it is necessary that the electron temperature should be larger than the ion temperature. If a flux tube suddenly expands, the ions will tend to cool adiabatically whereas the electrons will tend to remain at the temperature of the boundaries, since the electron thermal conductivity is much higher than the ion-thermal conductivity. Hence it is plausible that, as the result of a sudden eruption, an overlying flux tube could be disturbed in such a way that the ion temperature drops more than the electron temperature.

\section{Discussion}

We have seen that the mechanism for eruption of a filament and/or a CME is most likely due to an MHD instability, or a combined MHD-resistive instability. We have also seen that the main phase may plausibly be interpreted in terms of current interruption by the ion-acoustic instability, if a current-carrying flux tube is suddenly expanded and raised as the result of filament eruption. However, the proposed model raises many questions that require investigation. For instance, it will be necessary to determine whether the ion-acoustic instability is the most likely mechanism of current interruption. Also, we need to study the thermodynamic evolution of plasma in a suddenly erupting flux tube, to determine whether the ratio of electron temperature to ion temperature will indeed exceed unity by a significant factor. We need to determine the DC electric field that would develop in such a system and the particle distribution that would result from the combined action of the DC and oscillatory electric fields.

In addition, we need to inquire whether it is indeed possible to explain the observed properties of the solar corona on the assumption that there is no steady coronal heating, but only impulsive flare-like heating. Assuming that the spatial scale of this impulsive behavior is sub-telescopic, we need to consider a complete cycle in the life-history of a small flux tube, and then determine whether the time-averaged radiation and other properties of such a flux tube are consistent with the observed radiation and other properties of the corona. 


\section{Acknowledgements}

It is a pleasure to acknowledge helpful discussion on these topics with James Klimchuk and Taeil Bai and helpful comments on this article from B. C. Low. This work was supported by ONR through contract N00014-85-K-0111, and by NASA through grant NGL 05-020-272 and through the Solar-A collaboration under contract NAS8-37334 with Lockheed Palo Alto Research Laboratories.

\section{References}

Alfvén, H. and Carlqvist, P.: 1967, Solar Phys. 1, 220.

Aly, J. J.: 1984, Astrophys. J. 283, 349.

Anzer, U. and Pneuman, G. W.: 1982, Solar Phys. 79, 129.

Bai, T.: 1986a, Adv. Space Res. 6, 203.

Bai, T.: 1986b, Astrophys. J. 308, 912.

Bai, T. and Sturrock, P. A.: 1989, Ann. Rev. Astron. Astrophys. 27 (in press).

Bernstein, I. B.: 1973a, in M. N. Rosenbluth and R. Z. Sagdeev (eds.), Handbook of Plasma Physics.

Bernstein, I. B.: 1973b, in A. A. Galeev and R. N. Sudan (eds.), Basic Plasma Physics I, p. 421.

Blake, M. L. and Sturrock, P. A.: 1985, Astrophys. J. 290, 359.

Birn, J., Goldstein, H., and Schindler, K.: 1978, Solar Phys. 57, 81.

Cliver, E. W., Dennis, B. R., Kiplinger, A. L., Kane, S. R., Neidig, D. F., Sheeley, N. R., and Koomen, M. J.: 1986, Astrophys. J. 305, 920.

Kahler, S. W.: 1982, J. Geophys. Res. 87, 3439.

Kane, S. R., Chupp, E. L., Forrest, K. J., Share, G. H., and Rieger, E.: 1986, Astrophys. J. 300, L95.

Klimchuk, J. A. and Sturrock, P. A.: 1989, Astrophys. J. (in press).

Kopp, R. A. and Pneuman, G. W.: 1976, Solar Phys. 50, 85.

Krall, N. A. and Trivelpiece, A. W.: 1973, Principles of Plasma Physics, McGraw-Hill, New York, p. 475.

Low, B. C.: 1977a, Astrophys. J. 212, 234.

Low, B. C.: 1977b, Astrophys. J. 217, 988.

Low, B. C.: 1980, Astrophys. J. 239, 377.

Martin, S. F. and Ramsey, H. E.: 1972, in P. S. McIntosh and M. Dryer (eds.), Solar Activity Observations and Predictions, MIT Press, Cambridge, p. 371.

Parker, E. N.: 1988, Astrophys. J. 330, 474.

Priest, E. R. and Milne, A. M.: 1980, Solar Phys. 65, 315.

Sturrock, P. A.: 1968, in K. O. Kiepenheuer (ed.), 'Structure and Development of Solar Active Regions', IAU Symp. 35, 471.

Sturrock, P. A.: 1987, Solar Phys. 113, 13.

Sturrock, P. A.: 1988, Outstanding Problems in Solar System Plasma Physics, AGU Monograph (in press).

Sturrock, P. A., Kaufmann, P., Moore, R. L., and Smith, D. F.: 1984, Solar Phys. 94, 341.

Yang, W.-H., Sturrock, P. A., and Antiochos, S. K.: 1986, Astrophys. J. 309, 383. 